\title{
Editorial
}

\section{Legacy derived from Norin 10}

The international scientific community suffered a great loss with the sad passing of two wheat scientists who had made great contributions in wheat genetics and breeding. Prof. Mike Gale, former director of the John Innes Center in UK, died at the age of 65 on July 18, 2009 and Dr. Norman Borlaug, a Nobel Peace Prize laureate, died at the age of 95 on September 12, 2009. Although these two scientists had different interests and visions, it would be interesting to note that their scientific achievements were closely associated with Norin 10, a Japanese semi-dwarf wheat cultivar bred in Iwate Prefecture. Both will be remembered as pioneers of innovative researches involving Norin 10 semidwarfism loci that played significant roles in helping to feed the world's rapidly expanding population.

Dr. Norman Borlaug is also known as the father of the "Green Revolution" in recognition for his role in preventing world hunger and saving hundreds of millions of lives through his research on wheat in the 1950's and 1960's. Basically, he introduced the semidwarfism trait of Norin 10 to commonly cultivated wheat leading to the development of high-yielding varieties that eventually increased agricultural production at a time when people in Asia, Africa and South America were dying from hunger due to food shortage. In the recently published biography entitled "The Man Who Fed The World", the author, Leon Hesser provided a glimpse on how Dr. Borlaug fought against conservative breeders and politicians to advance his ideas on using the dwarfing trait to increase yield. With his success in developing high-yielding wheat varieties, he was able to show how innovative researches could provide the solution for solving world problems.

Prof. Mike Gale initiated a research program on genetic dwarfism in wheat, producing the first genetic map for the semidwarfing loci in the late 1970s and introducing the use of RFLP markers in genetic mapping of the wheat genome in 1980s. When the Rice Genome Research Program (RGP) was initiated in Japan in 1991, Mike, as colleagues and friends fondly addressed him, immediately started collaboration with RGP. With this collaboration, he elucidated the conservation in gene order in rice and wheat at the genetic level and literally concluded that "rice is wheat". His subse- quent studies on wheat semidwarfism derived from Norin 10 led to the molecular characterization of the Rht 1 gene. Using available genetic information from rice and Arabidopsis as well as partial cDNA nucleotide sequences for detailed mapping, he and his colleagues successfully identified the DELLA protein corresponding to the phenotype Rht 1 (Nature 400: 256-261, 1999).

These research achievements paved the way for major discoveries in rice research as well. It is well known that the works of Dr. Borlaug in wheat also led to the breeding of high-yielding semi-dwarf rice cultivars at the International Rice Research Institute in the 1970's. The comparative studies in rice and wheat spearheaded by Mike was extended to other crops thereby establishing the so-called "Crop Circle" model for the genetic alignment of all cereal genomes with rice as the reference genome. Characterization of the semidwarfing gene provided the characterization of phenotype slendar named by taller culm and encoding the same DELLA protein in rice at the corresponding locus of Rht1. Subsequently, Dr. Makoto Matsuoka of Nagoya University and his colleagues revealed that the slendar gene differs from $R h t 1$ as a result of a mutation in the region associated with gibberellins (GA) signaling. Further molecular analysis of mutants involved in GA signal pathway could identify GA binding protein, GID1, and recently three-dimensional structure of ternary complex of GA-DELLA-GID1 was elucidated. The impact of their discoveries has become apparent in other cereal crops with the development of many improved varieties.

There is no doubt both these two wheat scientists, Prof. Mike Gale and Dr. Norman Borlaug made significant contribution not only in breeding high yielding varieties but also in understanding the components for increasing yield. Their respective legacies, which were generated by studies using Norin 10 will continue to benefit research in cereal crops, as we face more challenges in increasing crop production for food security.

Takuji Sasaki Editor-in-chief National Institute of Agrobiological Sciences 